University of Wollongong

Research Online

Faculty of Engineering and Information

Faculty of Engineering and Information

Sciences - Papers: Part B

Sciences

2020

\title{
Beam-Based Analog Self-Interference Cancellation in Full-Duplex MIMO Systems
}

Anh Tuyen Le

AnhTuyen.Le@student.uts.edu.au

Le Chung Tran

University of Wollongong, Ictran@uow.edu.au

Xiaojing Huang

huang@uow.edu.au

Y. Jay Guo

Follow this and additional works at: https://ro.uow.edu.au/eispapers1

Part of the Engineering Commons, and the Science and Technology Studies Commons

Research Online is the open access institutional repository for the University of Wollongong. For further information contact the UOW Library: research-pubs@uow.edu.au 


\title{
Beam-Based Analog Self-Interference Cancellation in Full-Duplex MIMO Systems
}

\begin{abstract}
Self-interference (SI) cancellation for full-duplex (FD) multiple input multiple output (MIMO) systems is challenging due to both hardware and signal processing complexity. In this paper, a beam-based adaptive filter structure with analog least mean square (ALMS) loops is proposed to significantly reduce the complexity of SI cancellation for FD MIMO systems. With this structure, the number of adaptive filters required for SI cancellation scales linearly with the number of transmit beams rather than quadratically with the number of antennas. Furthermore, to avoid additional transmit chains used to upconvert the beam signals to generate reference signals for the ALMS loops, a novel method is proposed to select the optimized reference signals from all transmitted signals. In addition, our stationary analysis shows that the proposed structure for FD MIMO systems outperforms the ALMS loop employed for an FD single input single output system. Simulations are conducted to confirm the theoretical analyses.
\end{abstract}

\section{Keywords}

full-duplex, cancellation, self-interference, mimo, systems, analog, beam-based

Disciplines

Engineering | Science and Technology Studies

\section{Publication Details}

A. Le, L. Tran, X. Huang \& Y. Guo, "Beam-Based Analog Self-Interference Cancellation in Full-Duplex MIMO Systems," IEEE Transactions on Wireless Communications, vol. Online First, pp. 1-12, 2020. 


\title{
Beam-Based Analog Self-Interference
}

\section{Cancellation in Full-Duplex MIMO Systems}

\author{
Anh Tuyen Le, Le Chung Tran, Senior Member, IEEE, Xiaojing Huang, Senior \\ Member, IEEE, and Y. Jay Guo, Fellow, IEEE
}

\begin{abstract}
Self-interference (SI) cancellation for full-duplex (FD) multiple input multiple output (MIMO) systems is challenging due to both hardware and signal processing complexity. In this paper, a beambased adaptive filter structure with analog least mean square (ALMS) loops is proposed to significantly reduce the complexity of SI cancellation for FD MIMO systems. With this structure, the number of adaptive filters required for SI cancellation scales linearly with the number of transmit beams rather than quadratically with the number of antennas. Furthermore, to avoid additional transmit chains used to upconvert the beam signals to generate reference signals for the ALMS loops, a novel method is proposed to select the optimized reference signals from all transmitted signals. In addition, our stationary analysis shows that the proposed structure for FD MIMO systems outperforms the ALMS loop employed for an FD single input single output system. Simulations are conducted to confirm the theoretical analyses.
\end{abstract}

\section{Index Terms}

Full-duplex, self-interference cancellation, ALMS loop, beamforming, massive MIMO.

\section{INTRODUCTION}

A. T. Le, X. Huang and Y. Jay Guo are with Faculty of Engineering and IT, University of Technology Sydney, Ultimo, NSW, 2007, Australia (emails: anhtuyen.le@student.uts.edu.au, xiaojing.huang@uts.edu.au, and jay.guo@uts.edu.au).

L. C. Tran is with Faculty of Engineering and Information Sciences, University of Wollongong, Wollongong, NSW, 2522 Australia (email: lctran@uow.edu.au). 
7 HE next generation wireless communication systems will leap forward the data transmission performance in many aspects such as higher data rate, lower round-trip latency, and less power consumption [1]. To achieve these objectives, many disruptive technologies have been developed, including full-duplex (FD) and massive multiple input multiple output (MIMO) transmission [2], [3]. With FD operation, a transceiver can simultaneously transmit and receive signals in the same frequency band so that the throughput can be doubled compared to its halfduplex counterpart [4]. Massive MIMO is promising because it can support many users by using beamforming technique with increased spectral and energy efficiencies. Naturally, combining FD with massive MIMO can further improve spectral efficiency [5] and reduce the round-trip latency in networks requiring two-way relay channels [6].

A key issue that has prevented FD from becoming practical is the strong self-interference (SI) from the co-located transmitter, which blocks the receiver from the signal of interest. Therefore, investigation on SI mitigation has attracted significant attention from wireless industry and research community. It has been shown in the literature that the SI can be mitigated by consecutive passive suppression and active cancellation [7]. Passive suppression aims to isolate the transmitter and receiver in the propagation domain so that SI is attenuated [8]-[10]. Active cancellation intends to cancel the SI at both radio frequency (RF) domain [11]-[13] and digital domain [14], [15]. A combination of these SI mitigation schemes can suppress SI to noise floor level so that FD systems can be realized as demonstrated in [16], [17].

However, it is very challenging to mitigate SI in FD MIMO systems due to the complexity of the SI channels. In particular, in an $N \times N$ antenna FD MIMO system, at each receive (Rx) chain, there are presented SIs caused by the cross-talks from $N$ transmit (Tx) antennas. One advantage of MIMO systems is that beaforming techniques can be used to exploit spatial suppression in FD MIMO systems [18]-[23]. To further mitigate the level of SI, analog domain cancellation should be used. Among many different approaches, a multi-tap canceller has been proved as a promising structure for SI cancellation in FD single antenna systems [13], [24]-[26]. This is because the transmitted RF signal is utilized in this structure so that it can also mitigate the Tx noise and nonlinear distortions of the transmitter [27]. However, if a cancellation circuit is 
used for each pair of Tx and Rx antennas, an $N \times N$ antenna FD MIMO system will need $N^{2}$ cancellation circuits [24]. Therefore, some strategies of using analog cancellation circuits have been introduced in the literature to reduce the number of cancellers [24], [28]-[30]. However, the multi-tap structures used in these publications require channel state information (CSI) and digital signal processing (DSP) to synthesize the weighting coefficients in each tap. As a result, such FD MIMO has to operate in the half-duplex mode when estimating the SI channel. In addition, since the optimization depends on the cross-talk between each pair of co-located Tx and Rx antennas, the complexity of DSP algorithms increases quadratically with the size of FD MIMO systems [24]. The increase of DSP complexity results in larger digital hardware resources such as field programmable gate array (FPGA) and higher power consumption. As an example, DSP computations for 16 cancellation circuits in a $4 \times 4$ FD MIMO system will consume up to $80 \mathrm{~W}[24]$.

Unlike the multi-tap structures mentioned above, the analog least mean-square (ALMS) loop presented in [13] is an adaptive filter without any DSP involvement. Instead, a simple ResistorCapacitor (RC) low-pass filter (LPF) is utilized to synthesize the weighting coefficients for each tap. The behaviors of the ALMS loop in different FD single antenna systems have been presented in [13], [31]-[35]. A prototype of the ALMS loop has been demonstrated in [36]. Obviously, adopting the ALMS loop for SI cancellation in FD MIMO systems can significantly reduce the complexity of DSP at RF frontend.

In this paper, we propose a beam-based SI cancellation structure which employs ALMS loops as the adaptive filters for SI mitigation in FD massive MIMO systems. Since the transmitted signal power per antenna in FD massive MIMO systems is low, all Tx chains can be assumed to be linear. In this case, the SI at the receiver side can be considered as a linear combination of the transmitted beam signals for $K$ users. As a result, instead of using $N$ cancellers to cancel the SI from $N$ Tx antennas for each receive antenna, only $K$ adaptive filters are sufficient. Since $K \ll N$, the number of cancellation circuits is significantly reduced. Unlike the beam-based structure presented in [37] where additional Tx chains are employed to up-convert the beam signals to generate the required RF reference signals, in our proposed structure, the reference 
signals for the $K$ ALMS loops are selected from the $N$ transmitted signals. Hence, hardware complexity is significantly reduced. Stationary analysis is then applied to evaluate the interference suppression ratio given by the proposed cancellation structure. Further proposing a method to select a set of optimized reference signals, we show that the performance of the proposed beambased cancellation structure is superior to that of the structure with $N^{2}$ repeated ALMS loops for all Tx-Rx pairs. The contributions of this paper are summarized as follows.

- A mathematical model of the SI in FD beamforming MIMO systems is derived, which shows that the SI to each receive antenna is a linear combination of the transmitted beam signals. This model suggests that the reference signals can be the linear combinations of the beam signals, which can be selected from the Tx antennas. Hence no extra RF chain is required to produce the reference signals from the beam signals and the hardware complexity is significantly reduced.

- By employing the ALMS loop which does not require the CSI for tuning its weighting coefficients, the proposed structure can save significant FPGA resources and hence reduce energy consumption.

- A novel method is proposed to select the reference signals for the ALMS loops from available transmitted signals, which not only reduces the hardware complexity, but also enhances the level of cancellation given by the ALMS loops.

The rest of this paper is organized as follows. The proposed beam-based cancellation architecture for FD MIMO systems and signal models are described in Section II. In Section III, stationary analysis is presented to evaluate the performance of the proposed structure, and the method to select the reference signals is also devised. In Section IV, simulations are conducted to verify the theoretical analysis. Finally, conclusions are drawn in Section V.

Notation: We use boldface lowercase and boldface capital letters (e.g. a and A) to indicate vectors and matrices, respectively. $\mathbf{A}^{T}$ and $\mathbf{A}^{H}$ denote the transpose and Hermitian transpose of A, respectively, while $\otimes$ is the Kronecker product. We denote $\operatorname{diag}\left\{\lambda_{i}\right\}, i=1, \cdots, M K L$, as an $M \times K \times L$ diagonal matrix whose main diagonal elements are $\lambda_{1}, \cdots, \lambda_{M K L} . \mathbf{I}_{M}$ represents the identity matrix of order $M . E\{$.$\} stands for the ensemble expectation operation, and \mathbb{C}$ is 


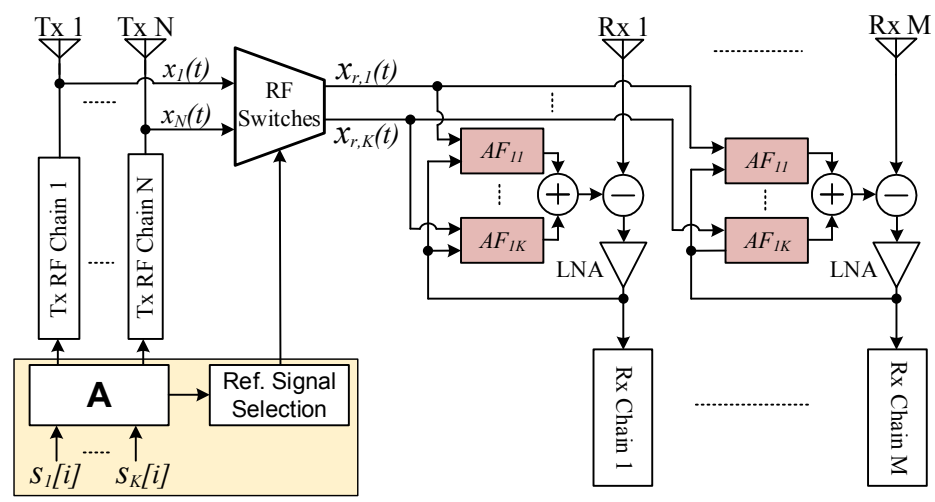

Fig. 1. Proposed beam-based analog SI cancellation structure.

the complex set.

\section{SYSTEM DESCRIPTIONS}

\section{A. Beam-Based Analog SI Cancellation}

Consider an FD MIMO digital beamforming system with $N \mathrm{Tx}$ and $M \mathrm{Rx}$ antennas as shown in Fig. 1. The transmitted data symbols for $K$ directions, denoted as $s_{k}(i), i=-\infty, \ldots, \infty, k=$ $1, \ldots, K$, are multiplied with the corresponding array vector $\mathbf{a}\left(\theta_{k}\right)$ before added together and transmitted by $N$ Tx chains. The vector of RF transmitted signals at $N$ antennas is expressed as

$$
\mathbf{x}(t)=\operatorname{Re}\left\{\mathbf{X}(t) e^{j 2 \pi f_{c} t}\right\}
$$

where $\mathbf{X}(t)=\left[\begin{array}{llll}X_{1}(t) & \cdots & X_{N}(t)\end{array}\right]^{T}$ is the baseband equivalent vector. $\mathbf{X}(t)$ can be expressed as

$$
\mathbf{X}(t)=\mathbf{A S}(t)=\left[\begin{array}{ccc}
a_{0}\left(\theta_{1}\right) & \cdots & a_{0}\left(\theta_{K}\right) \\
\vdots & \ddots & \vdots \\
a_{N-1}\left(\theta_{1}\right) & \cdots & a_{N-1}\left(\theta_{K}\right)
\end{array}\right]\left[\begin{array}{c}
S_{1}(t) \\
\vdots \\
S_{K}(t)
\end{array}\right]
$$

where $a_{n}\left(\theta_{k}\right)=e^{j n \frac{2 \pi}{\lambda} d \sin \theta_{k}}, n=0, \ldots, N-1$, is the array vector of the beam at direction $\theta_{k}, d$ is the distance between antenna elements, $S_{k}(t)=\sum_{i=-\infty}^{\infty} s_{k}(i) p\left(t-i T_{s}\right)$ is the low-pass equivalent of the transmitted signal for the $k$-th user, $s_{k}(i)$ is the complex data symbol whose interval is 
$T_{s}$, and $p(t)$ is the pulse shaping filter. In the rest of this paper, the following assumptions are applied.

1) Transmit data for all directions are independent, i.e.,

$$
E\left\{s_{k}(i)^{*} s_{k^{\prime}}\left(i^{\prime}\right)\right\}=\left\{\begin{array}{l}
1, \text { for } k=k^{\prime} \text { and } i=i^{\prime} \\
0, \text { for } k \neq k^{\prime} \text { or } i \neq i^{\prime}
\end{array} .\right.
$$

2) All Tx and Rx chains are linear and have the same configuration. The gain of the power amplifier is absorbed into the pulse shaping filter.

Due to the FD operation, at the inputs of $M \mathrm{Rx}$ chains, there are $M$ SI signals caused by $N$ transmitted antennas, denoted as $\mathbf{z}(t)=\operatorname{Re}\left\{\mathbf{Z}(t) e^{j 2 \pi f_{c} t}\right\}=\operatorname{Re}\left\{\left[Z_{1}(t), \ldots, Z_{M}(t)\right]^{T} e^{j 2 \pi f_{c} t}\right\}$. Assuming that the SI channel between a pair of co-located Tx and Rx antennas includes $L$ paths, and the transmitted signal in the $l$-th path is delayed by $l T_{d}, l=0, \ldots, L-1$, where $T_{d}$ is the time delay between two adjacent paths. The baseband equivalents of all of the SI signals can be represented as

$$
\mathbf{Z}(t)=\left[Z_{1}(t) \cdots Z_{M}(t)\right]^{T}=\sum_{l=0}^{L-1} \mathbf{H}^{H}(l) \mathbf{A} \mathbf{S}\left(t-l T_{d}\right)
$$

where $\mathbf{H}(l)=\left[\begin{array}{ccc}h_{1,1}(l) & \cdots & h_{1, M}(l) \\ \vdots & \ddots & \vdots \\ h_{N, 1}(l) & \cdots & h_{N, M}(l)\end{array}\right]$ is the $N \times M$ coefficient matrix at the $l$-th path of all SI channels.

It can be seen from (4) that the vector $\mathbf{Z}(t)$ is a linear combination of all basis vectors $\mathbf{S}\left(t-l T_{d}\right), l=0, \ldots, L-1$. The concept of beam-based cancellation is that the vector of cancellation signals can be obtained by a linear transformation of the same basis $\mathbf{S}\left(t-l T_{d}\right), l=$ $0, \ldots, L-1$. This concept leads to two important points in the construction of the cancellation structure. Firstly, multi-tap adaptive filters to approximate all the SI channels should be used. Secondly, the reference signals for the cancellation circuit should be generated from the $K$ beam signals. As illustrated in Fig. 1 , at the input of the $m$-th $\mathrm{Rx}$ chain, $K$ adaptive filters $\left(A F_{m, k}, k=\right.$ $1, \ldots, K)$ are employed to correspondingly modify the $K$ reference signals $x_{r, k}(t), k=1, \ldots, K$. 


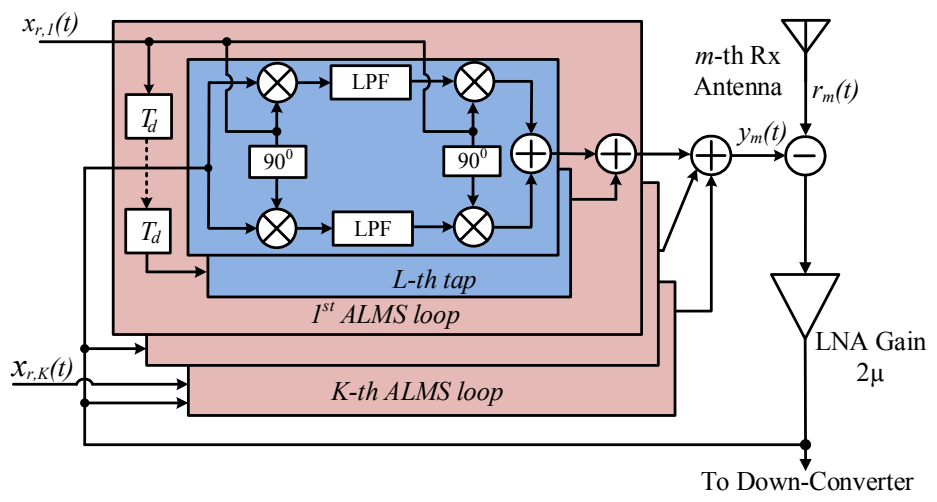

Fig. 2. ALMS loop for beam-based cancellation structure.

The outputs of the $K$ adaptive filters are added together to generate the cancellation signal $y_{m}(t), M=1, \cdots, M$. If each adaptive filter also has $L$ taps with the tap delay $T_{d}$, the vector of cancellation signals $\mathbf{y}(t)=\left[y_{1}(t), \ldots, y_{M}(t)\right]^{T}$ is represented by

$$
\mathbf{y}(t)=\operatorname{Re}\left\{\sum_{l=0}^{L-1} \mathbf{W}^{H}(l, t) \mathbf{x}_{r}\left(t-l T_{d}\right)\right\}
$$

where $\mathbf{W}(l, t)=\left[\begin{array}{ccc}w_{1,1}(l, t) & \cdots & w_{1, M}(l, t) \\ \vdots & \ddots & \vdots \\ w_{K, 1}(l, t) & \cdots & w_{K, M}(l, t)\end{array}\right]$ is the weighting coefficient matrix at the $l$-th tap of all adaptive filters, and $\mathbf{x}_{r}(t)=\left[x_{r, 1}(t), \ldots, x_{r, K}(t)\right]^{T}$ is the vector of reference signals, which can be generated from beam signals $s_{k}(i), k=1, \ldots, K$, in several ways. The easiest way is employing additional Tx chains which are the same as those in the main chains to up-convert and amplify the beam signals as in [37]. However, this leads to more hardware complexity. Another way to obtain $\mathbf{x}_{r}(t)$ is to select them from $N$ transmitted signals as shown in Fig. 1. The method to select the optimized reference signals will be discussed in the next section.

\section{B. ALMS Loop}

As mentioned in the Introduction, all adaptive filters can be implemented by ALMS loops as presented in Fig. 2. From the concept of beam-based cancellation, at the $m$-th Rx chain, $K$ ALMS loops are employed to generate the cancellation signal. Operation of the ALMS loop is described 
as follows. Each ALMS loop has $L$ taps in which each tap includes a tap delay, a quadrature demodulator, LPFs, and a quadrature modulator. The ALMS loop has two inputs for the reference and the looped-back signals and one output for the cancellation signal. For simplicity, the losses caused by power splitters for both reference and looped-back signals are normalized to one. Considering the $k$-th ALMS loop, at the $l$-th tap, the delayed reference signal $\left.x_{r, k}(t-l) T_{d}\right), l=$ $0, \cdots, L-1$, is multiplied by the looped-back signal at the demodulator. The output of this demodulator is then filtered by the RC LPF to obtain the weighting coefficients $w_{k, m}(l, t)$ which will modulate a copy of the delayed reference signal $x_{r, k}\left(t-l T_{d}\right)$ at the modulator. The outputs of all $L$ taps are added together before combined with the outputs of other ALMS loops to generate the cancellation signal $y_{m}(t)$ which is used to cancel the SI $z_{m}(t)$ at the input of the $m$-th $\mathrm{Rx}$ chain. The residual signal is amplified and looped-back to the inputs of all ALMS loops.

The receive signal at the $m$-th $\mathrm{Rx}$ chain, denoted as $r_{m}(t)$, includes the desired signal from the remote end $s_{m, r}(t)$, the SI from $N$ co-located transmitters $z_{m}(t)$ and the additive Gaussian noise $n_{m}(t)$, i.e., $r_{m}(t)=z_{m}(t)+s_{m, r}(t)+n_{m}(t)=\operatorname{Re}\left\{\left[Z_{m}(t)+S_{m, r}(t)+N_{m}(t)\right] e^{j 2 \pi f_{c} t}\right\}$ where $Z_{m}(t), S_{m, r}(t)$ and $N_{m}(t)$ are the low-pass equivalents of these signals, respectively. Without loss of generality, we assume that the transmitted data $S_{k}(t), k=1, \ldots, K, S_{m, r}(t)$, and $N_{m}(t), m=$ $1, \ldots, M$, are all independent. From [13, Eq. (5)], the vector of weighting coefficients at the $m$-th $\mathrm{Rx}$ chain $\mathbf{w}_{m}(l, t)=\left[w_{1, m}(l, t), \ldots, w_{K, m}(l, t)\right]^{T}, l=0, \ldots, L-1$, is obtained by

$$
\mathbf{w}_{m}\left(l, t+t_{0}\right)=\frac{2 \mu \alpha}{K_{1} K_{2}} \int_{t_{0}}^{t+t_{0}} e^{-\alpha(t-\tau)}\left[r_{m}(\tau)-y_{m}(\tau)\right] \mathbf{X}_{r}\left(\tau-l T_{d}\right) e^{j 2 \pi f_{c}\left(\tau-l T_{d}\right)} d \tau
$$

where $K_{1}$ and $K_{2}$ are the dimensional constants of the multipliers inside the demodulators and modulators, respectively, $2 \mu$ is the gain of the LNA, $\alpha=1 / R C$ is the decay constant of the LPF with resistance $R$ and capacitance $C, \mathbf{X}_{r}(t)$ is the vector of baseband equivalents of the reference signals, and $0 \leq t_{0}<T_{s}$ is an initial starting time. 


\section{StATIONARY ANALYSis}

In this section, we apply stationary analysis to analyze the performance of the proposed structure. Strictly speaking, the signals in a digital communication system demonstrate cyclostationary properties [13]. Stationary analysis performs time averaging on the signals over one symbol duration $T_{s}$ so that the system performance at macro scale can be tractably evaluated. Therefore, for stationary analysis, both ensemble expectation and time averaging (over one $T_{s}$ ) operations, denoted as $\bar{E}\{$.$\} , are applied to evaluate the random processes. From the assumptions in Section$ II, the normalized auto-correlation functions of the transmitted signals $S_{k}(t), k=1, \ldots, K$, are defined as

$$
\Phi_{k, k^{\prime}}(\tau)=\frac{1}{K_{1} K_{2}} \bar{E}\left\{S_{k}^{*}(t) S_{k^{\prime}}(t-\tau)\right\}= \begin{cases}\Phi(\tau) & \text { for } k=k^{\prime} \\ 0 & \text { for } k \neq k^{\prime}\end{cases}
$$

where $\Phi(\tau)=\frac{1}{K_{1} K_{2} T_{s}} \int_{0}^{T_{s}} p^{*}(t) p(t-\tau) d t$

\section{A. Cancellation Performance}

The cancellation performance of the proposed structure can be presented by the interference suppression ratio (ISR) which is the ratio between the total residual SI power $P_{\mathbf{d}}(t)$ after cancellation and the total SI power $P_{\mathrm{z}}$ across all $\mathrm{Rx}$ chains. Denoting the residual signal at the $m$-th Rx chain as $d_{m}(t)=z_{m}(t)-y_{m}(t)$, ISR is defined as

$$
\operatorname{ISR}(t)=\frac{P_{\mathbf{d}}(t)}{P_{\mathbf{z}}}
$$

where $P_{\mathbf{d}}(t)=\sum_{m=1}^{M} P_{d_{m}}(t)$ and $P_{\mathbf{z}}=\sum_{m=1}^{M} P_{z_{m}}$. In this evaluation, we evaluate the performance of the proposed structure under one realization of the SI channels but with random transmitted data symbols. Therefore, $P_{\mathbf{z}}$ does not change over the time. Meanwhile, the residual SI power is a function of time to show the convergence property of all ALMS loops. When the SI channels vary, the weighting coefficients of all ALMS loops will adapt accordingly. To derive $I S R(t), P_{\mathbf{z}}$ and $P_{\mathbf{d}}(t)$ are calculated as follows. 
1) Total SI power: In general, the SI signal at the $m$-th $\mathrm{Rx}$ chain can be expressed as

$$
Z_{m}(t)=\sum_{n=1}^{N} \int_{-\infty}^{\infty} h_{n, m}^{*}(\tau) X_{n}(t-\tau) d \tau
$$

where $h_{n, m}(\tau)$ is the actual SI channel between the $n$-th Tx and $m$-th $\mathrm{Rx}$ antenna. The total normalized (to $K_{1} K_{2}$ ) SI power across all $M \mathrm{Rx}$ chains is calculated as

$$
\begin{aligned}
P_{\mathbf{z}} & =\sum_{m=1}^{M} \frac{1}{K_{1} K_{2}} \bar{E}\left\{z_{m}^{2}(t)\right\}=\sum_{m=1}^{M} \frac{1}{2 K_{1} K_{2}} \bar{E}\left\{\left|Z_{m}(t)\right|^{2}\right\} \\
& =\frac{1}{2 K_{1} K_{2}} \sum_{m=1}^{M} \sum_{n=1}^{N} \sum_{n^{\prime}=1}^{N} \int_{-\infty}^{\infty} \int_{-\infty}^{\infty} h_{n, m}^{*}(\tau) \bar{E}\left\{X_{n}(t-\tau) X_{n^{\prime}}^{*}\left(t-\tau^{\prime}\right)\right\} h_{n^{\prime}, m}\left(\tau^{\prime}\right) d \tau d \tau^{\prime} \\
& =\frac{1}{2 K_{1} K_{2}} \sum_{m=1}^{M} \sum_{n=1}^{N} \sum_{n^{\prime}=1}^{N} \int_{-\infty}^{\infty} \int_{-\infty}^{\infty} h_{n, m}^{*}(\tau) \Phi\left(\tau-\tau^{\prime}\right) \mathbf{a}_{n} \mathbf{a}_{n^{\prime}}^{H} h_{n^{\prime}, m}\left(\tau^{\prime}\right) d \tau d \tau^{\prime}
\end{aligned}
$$

where $\mathbf{a}_{n}$ is the $n$-th row of the matrix $\mathbf{A}$.

The ALMS loop is designed based on the assumption that the SI channel can be approximated as a $L$-stage filter, i.e.,

$$
Z_{m}(t)=\sum_{n=1}^{N} \int_{-\infty}^{\infty} h_{n, m}^{*}(\tau) X_{n}(t-\tau) d \tau \approx \sum_{n=1}^{N} \sum_{l=0}^{L-1} h_{n, m}^{*}(l) X_{n}\left(t-l T_{d}\right)
$$

Therefore, the vector $h_{n, m}(l), l=0, \ldots, L-1, n=1, \ldots, N$, can be found by minimizing the following modeling error

$$
\epsilon_{m}^{2}=\frac{1}{K_{1} K_{2}} \bar{E}\left\{\left|\sum_{n=1}^{N} \int_{-\infty}^{\infty} h_{n, m}^{*}(\tau) X_{n}(t-\tau) d \tau-\sum_{n=1}^{N} \sum_{l=0}^{L-1} h_{n, m}^{*}(l) X_{n}\left(t-l T_{d}\right)\right|^{2}\right\}
$$

Geometrically, $X_{n}\left(t-l T_{d}\right), n=1, \cdots, N$, and $l=0, \cdots, L-1$, can be seen as an orthogonal basis for a space $\mathbb{C}^{N \times L}$. From the principle of orthogonality, the modeling error is minimized when $h_{n, m}^{*}(l) X_{n}\left(t-l T_{d}\right)$ are the projections of $Z_{m}(t)$ on $\mathbb{C}^{N \times L}$, i.e.,

$$
\bar{E}\left\{X_{n}\left(t-l T_{d}\right)\left[\sum_{n^{\prime}=1}^{N} \int_{-\infty}^{\infty} h_{n^{\prime}, m}(\tau) X_{n^{\prime}}^{*}(t-\tau) d \tau-\sum_{n^{\prime}=1}^{N} \sum_{l^{\prime}=0}^{L-1} h_{n^{\prime}, m}\left(l^{\prime}\right) X_{n^{\prime}}^{*}\left(t-l^{\prime} T_{d}\right)\right]\right\}=0
$$

for $n=1, \ldots, N$ and $l=0, \ldots, L-1$. After some multiplications and extensions for $M \mathrm{Rx}$ 
chains (see Appendix A), we find the vector of modeled channel coefficients $\mathbf{h}=\left[h_{1,1}(0), \ldots h_{1,1}(L-\right.$ $\left.1), \ldots h_{N, M}(0) \ldots h_{N, M}(L-1)\right]^{T}$ as

$$
\mathbf{h}=\left[\mathbf{I}_{N M} \otimes \boldsymbol{\Phi}\right]^{-1}\left[\begin{array}{c}
\int_{-\infty}^{\infty} \Phi(-\tau) h_{1,1}(\tau) d \tau \\
\vdots \\
\int_{-\infty}^{\infty} \Phi\left((L-1) T_{d}-\tau\right) h_{N, M}(\tau) d \tau
\end{array}\right]
$$

where $\Phi=\left[\begin{array}{cccc}\Phi(0) & \Phi\left(-T_{d}\right) & \cdots & \Phi\left(-(L-1) T_{d}\right) \\ \vdots & \vdots & \ddots & \vdots \\ \Phi\left((L-1) T_{d}\right) & \Phi\left((L-2) T_{d}\right) & \cdots & \Phi(0)\end{array}\right]$ is the auto-correlation matrix of the transmitted signals. Therefore, when calculating the signal power using the SI channel model, we need to take into account the total modeling error, denoted as $\epsilon$, derived in Appendix A as

$$
\epsilon^{2}=\sum_{m=1}^{M} \epsilon_{m}^{2}=\sum_{m=1}^{M} \sum_{n=1}^{N} \sum_{n^{\prime}=1}^{N} \int_{-\infty}^{\infty} \int_{-\infty}^{\infty} h_{n, m}^{*}(\tau) \Phi\left(\tau-\tau^{\prime}\right) \mathbf{a}_{n} \mathbf{a}_{n^{\prime}}^{H} h_{n^{\prime}, m}\left(\tau^{\prime}\right) d \tau d \tau^{\prime}-\underline{\mathbf{h}}^{H}\left[\mathbf{I}_{M} \otimes \boldsymbol{\Theta}\right] \underline{\mathbf{h}}
$$

where $\underline{\mathbf{h}}=\left[\mathbf{I}_{M} \otimes\left(\mathbf{A}^{H} \otimes \mathbf{I}_{L}\right)\right] \mathbf{h}$, and $\boldsymbol{\Theta}=\mathbf{I}_{K} \otimes \boldsymbol{\Phi}$. Hence, the total normalized SI power at all $M \mathrm{Rx}$ chains can also be calculated as

$$
P_{\mathbf{z}}=\frac{1}{2} \epsilon^{2}+\frac{1}{2} \underline{\mathbf{h}}^{H}\left[\mathbf{I}_{M} \otimes \boldsymbol{\Theta}\right] \underline{\mathbf{h}} .
$$

2) Residual SI power: Following the same steps above, the power of the residual SI at the $m$-th Rx chain can be calculated as

$$
\begin{aligned}
& P_{d_{m}}(t)=\frac{1}{K_{1} K_{2}} \bar{E}\left\{\left[z_{m}(t)-y_{m}(t)\right]^{2}\right\}=\frac{1}{2 K_{1} K_{2}} \bar{E}\left\{\left|Z_{m}(t)-Y_{m}(t)\right|^{2}\right\} \\
& =\frac{1}{2 K_{1} K_{2}} \bar{E}\left\{\mid\left[Z_{m}(t)-\sum_{l=0}^{L-1} \mathbf{h}_{m}^{H}(l) \mathbf{X}\left(t-l T_{d}\right)\right]+\right. \\
& \left.\left.\sum_{l=0}^{L-1}\left[\mathbf{h}_{m}^{H}(l) \mathbf{X}\left(t-l T_{d}\right)-\mathbf{w}_{m}^{H}(l, t) e^{-j 2 \pi f_{c} l T_{d}} \mathbf{X}_{r}\left(t-l T_{d}\right)\right]\right|^{2}\right\}
\end{aligned}
$$

where $\mathbf{h}_{m}(l), \mathbf{w}_{m}(l)$ are the $m$-th column vectors of the matrices $\mathbf{H}(l), \mathbf{W}(l)$, respectively, $\mathbf{X}_{r}(t)=\mathbf{A}_{r} \mathbf{S}(t)$ is the low-pass equivalent of $K$ reference signals selected from $\mathbf{X}(t)$, and 
$\mathbf{A}_{r} \subset \mathbf{A}$ is a $K \times K$ matrix obtained from $\mathbf{A}$. Since $\mathbf{X}_{r}(t)$ is a subset of $\mathbf{X}(t)$, from the principle of orthogonality, $P_{d_{m}}(t)$ can be rewritten as

$$
P_{d_{m}}(t)=\frac{1}{2} \epsilon_{m}^{2}+\frac{1}{2 K_{1} K_{2}} \bar{E}\left\{\left|\sum_{l=0}^{L-1}\left[\mathbf{h}_{m}^{H}(l) \mathbf{A}-\mathbf{w}_{m}^{H}(l, t) e^{-j 2 \pi f_{c} l T_{d}} \mathbf{A}_{r}\right] \mathbf{S}\left(t-l T_{d}\right)\right|^{2}\right\} .
$$

Denote $\mathbf{u}_{m}(l, t)=\left[u_{m, 1}(l, t), \ldots, u_{m, K}(l, t)\right]^{T}=\mathbf{A}^{H} \mathbf{h}_{m}(l)-\mathbf{A}_{r}^{H} \mathbf{w}_{m}(l, t) e^{j 2 \pi f_{c} l T_{d}}$ as the vector of weighting error functions, which shows the difference between the channel coefficients of the $l$-th tap in the SI channel and the weighting coefficients of the same tap in the $K$ ALMS loops at the $m$-th $\mathrm{Rx}$ chain. The expression of $P_{d_{m}}(t)$ becomes

$$
\begin{aligned}
& P_{d_{m}}(t)=\frac{1}{2} \epsilon_{m}^{2}+\frac{1}{2 K_{1} K_{2}} \bar{E}\left\{\left|\sum_{l=0}^{L-1} \mathbf{u}_{m}^{H}(l, t) \mathbf{S}\left(t-l T_{d}\right)\right|^{2}\right\} \\
& =\frac{1}{2} \epsilon_{m}^{2}+\frac{1}{2 K_{1} K_{2}} \bar{E}\left\{\sum_{l=0}^{L-1} \mathbf{u}_{m}^{H}(l, t) \mathbf{S}\left(t-l T_{d}\right) \sum_{l^{\prime}=0}^{L-1} \mathbf{S}^{H}\left(t-l^{\prime} T_{d}\right) \mathbf{u}_{m}\left(l^{\prime}, t\right)\right\} \\
& =\frac{1}{2} \epsilon_{m}^{2}+\frac{1}{2} \sum_{l=0}^{L-1} \sum_{\substack{l^{\prime}=0 \\
l^{\prime} \neq l}}^{L-1} \overline{\overline{\mathbf{u}}}_{m}^{H}(l, t) \Theta\left(\left(l-l^{\prime}\right) T_{d}\right) \overline{\overline{\mathbf{u}}}_{m}\left(l^{\prime}, t\right)+\frac{1}{2} \Phi(0) \sum_{l=0}^{L-1} \sum_{k=1}^{K} \overline{\bar{u}}_{m, k}^{2}(l, t) \\
& =\frac{1}{2} \epsilon_{m}^{2}+\frac{1}{2} \overline{\overline{\mathbf{u}}}_{m}^{H}(t) \Theta \overline{\overline{\mathbf{u}}}_{m}(t)+\frac{1}{2} \Phi(0)\left[\sum_{l=0}^{L-1} \sum_{k=1}^{K} \overline{\bar{u}}_{m, k}^{2}(l, t)-\overline{\overline{\mathbf{u}}}_{m}^{H}(t) \overline{\overline{\mathbf{u}}}_{m}(t)\right]
\end{aligned}
$$

where $\overline{\overline{\mathbf{u}}}_{m}(l, t)=\bar{E}\left\{\mathbf{u}_{m}(l, t)\right\}$ and $\overline{\bar{u}}_{m, k}^{2}(l, t)=\bar{E}\left\{u_{m, k}^{2}(l, t)\right\}$. Hence, the total residual SI power of all $M \mathrm{Rx}$ chains is represented by

$$
\begin{aligned}
P_{\mathbf{d}}(t) & =\sum_{m=1}^{M} P_{d_{m}}(t) \\
& =\frac{1}{2} \epsilon^{2}+\frac{1}{2} \overline{\overline{\mathbf{u}}}^{H}(t)\left[\mathbf{I}_{M} \otimes \boldsymbol{\Theta}\right] \overline{\overline{\mathbf{u}}}(t)+\frac{1}{2} \Phi(0)\left[\sum_{m, k, l} \overline{\bar{u}}_{m, k}^{2}(l, t)-\overline{\overline{\mathbf{u}}}^{H}(t) \overline{\overline{\mathbf{u}}}(t)\right]
\end{aligned}
$$

where $\overline{\overline{\mathbf{u}}}(t)=\left[\overline{\bar{u}}_{1,1}(0, t), \ldots, \overline{\bar{u}}_{1,1}(L-1, t), \ldots, \overline{\bar{u}}_{M, K}(L-1, t)\right]^{T}$. Therefore, we need to derive the weighting error function vector $\overline{\overline{\mathbf{u}}}(t)$ and $\sum_{m, k, l} \overline{\bar{u}}_{m, k}^{2}(l, t)$ to calculate the residual SI power.

Substituting the definition of the weighting error function into (6), after some multiplications (see Appendix B), we have the equation that the expected weighting error function vector should 
satisfy

$$
\overline{\overline{\mathbf{u}}}(t)=\underline{\mathbf{h}}-\mu \alpha \int_{0}^{t} e^{-\alpha(t-\tau)} \boldsymbol{\Psi} \overline{\overline{\mathbf{u}}}(\tau) d \tau
$$

where $\boldsymbol{\Psi}=\mathbf{I}_{M} \otimes\left[\left(\mathbf{B} \otimes \mathbf{I}_{L}\right) \boldsymbol{\Theta}\right]$ with $\mathbf{B}=\mathbf{A}_{r}^{H} \mathbf{A}_{r}$. We can see that (21) has the same form as that in the single antenna case [13, Eq. (26)]. Following similar steps shown in [13], we obtain the solution of the expected the weighting error function vector as

$$
\begin{aligned}
& \overline{\overline{\mathbf{u}}}(t)=\mathbf{Q} \operatorname{diag}\left\{\frac{1}{1+\mu \lambda_{i}}+\frac{\mu \lambda_{i}}{1+\mu \lambda_{i}} e^{-\left(1+\mu \lambda_{i}\right) \alpha t}\right\} \mathbf{Q}^{-1} \underline{\mathbf{h}}, \\
& \sum_{m, k, l} \overline{\bar{u}}_{m, k}^{2}(l, t)=\underline{\mathbf{h}}^{H} \mathbf{Q} \operatorname{diag}\left\{\frac{1}{\left(1+\mu \lambda_{i}\right)^{2}}+\frac{2 \mu \lambda_{i}}{\left(1+\mu \lambda_{i}\right)^{2}} \times\right. \\
& \left.e^{-\left(1+\mu \lambda_{i}\right) \alpha t}+\frac{\left(\mu \lambda_{i}\right)^{2} e^{-2\left(1+\mu \lambda_{i}\right) \alpha t}}{\left(1+\mu \lambda_{i}\right)^{2}}-e^{-2(1+\mu \bar{\lambda}) \alpha t}\right\} \mathbf{Q}^{-1} \underline{\mathbf{h}}
\end{aligned}
$$

where $\lambda_{i}, i=1, \ldots, M \times K \times L$, are the eigenvalues of $\Psi$ and $\mathbf{Q}$ is the orthonormal modal matrix whose columns are the $M \times K \times L$ eigenvectors of $\boldsymbol{\Psi}$, and $\bar{\lambda}=\Phi(0)$.

From (16) and (20), we obtain the ISR as

$$
\begin{aligned}
I S R(t) & =\frac{P_{\mathbf{d}}(t)}{P_{\mathbf{z}}} \\
& =\frac{\epsilon^{2}+\overline{\overline{\mathbf{u}}}^{H}(t)\left[\mathbf{I}_{M} \otimes \boldsymbol{\Theta}\right] \overline{\overline{\mathbf{u}}}(t)+\bar{\lambda}\left[\sum_{m, k, l} \overline{\bar{u}}_{m, k}^{2}(l, t)-\overline{\overline{\mathbf{u}}}^{H}(t) \overline{\overline{\mathbf{u}}}(t)\right]}{\epsilon^{2}+\underline{\mathbf{h}}^{H}\left[\mathbf{I}_{M} \otimes \boldsymbol{\Theta}\right] \underline{\mathbf{h}}} .
\end{aligned}
$$

Eq. (23) represents the performance of the proposed structure at time instance $t$. We can see that when $t \gg \frac{1}{\alpha(1+\mu \bar{\lambda})}$, all the ALMS loops have converged to their stable state. In this case, $\overline{\overline{\mathbf{u}}}(t) \rightarrow \overline{\overline{\mathbf{u}}}=\mathbf{Q} \operatorname{diag}\left\{\frac{1}{1+\mu \lambda_{i}}\right\} \mathbf{Q}^{-1} \underline{\mathbf{h}}$ and $\sum_{m, k, l} \overline{\bar{u}}_{m, k}^{2}(l, t) \rightarrow \overline{\overline{\mathbf{u}}}^{H} \overline{\overline{\mathbf{u}}}$, (23) becomes

$$
\begin{aligned}
I S R & =\left.I S R(t)\right|_{t \gg \frac{1}{\alpha(1+\mu \lambda)}} \\
& =\frac{\epsilon^{2}+\overline{\overline{\mathbf{u}}}^{H}\left[\mathbf{I}_{M} \otimes \boldsymbol{\Theta}\right] \overline{\overline{\mathbf{u}}}}{\epsilon^{2}+\underline{\mathbf{h}}^{H}\left[\mathbf{I}_{M} \otimes \boldsymbol{\Theta}\right] \underline{\mathbf{h}}} .
\end{aligned}
$$

To study the behavior of the proposed structure, we consider some special cases as follows.

1) When $M=N=K=1$ corresponding to an FD single antenna system, (24) becomes [13, Eq. (39)]. It means that the ALMS loop in the FD single antenna system is a special 
case of this beam-based cancellation structure. Hence, the properties of the ALMS loop presented in [13], [31], [32], [34], [35], such as the interference suppression lower bound and convergence speed, are also applicable in this case.

2) When $\mathbf{B}=\mathbf{I}_{K}$, i.e., additional Tx chains are employed to up-convert $K$ beam signals to form RF reference signals, $\Psi=\mathbf{I}_{M} \otimes \Theta=\mathbf{I}_{M K} \otimes \boldsymbol{\Phi}$, (24) becomes

$$
I S R=\frac{\epsilon^{2}+\underline{\mathbf{h}}^{H} \mathbf{Q} \operatorname{diag}\left\{\frac{\lambda_{i}}{\left(1+\mu \lambda_{i}\right)^{2}}\right\} \mathbf{Q}^{-1} \underline{\mathbf{h}}}{\epsilon^{2}+\underline{\mathbf{h}}^{H} \mathbf{Q} \operatorname{diag}\left\{\lambda_{i}\right\} \mathbf{Q}^{-1} \underline{\mathbf{h}}} .
$$

From the property of the Kronecker product, since the eigenvalues of $\mathbf{I}_{M K}$ are all one, $\lambda_{i}, i=1, \ldots, M K L$, are $M \times K$ replica of the eigenvalues of $\boldsymbol{\Phi}$. Therefore, (25) gives the same result as [13, Eq. (39)] in single antenna systems. It means that if additional Tx chains were employed to generate the reference signals for the $M \times K$ ALMS loops, the SI cancellation performance would be the same as that of the ALMS loop in a single antenna FD system.

Since no additional Tx chain is employed in this structure, a new factor affecting its performance is how to select the reference signals from $N$ transmitted signals. This problem is addressed as follows.

\section{B. Selection of Reference Signals}

In this section, we present an algorithm to select the reference signals for the adaptive filters. From (24), the performance of the proposed structure can be represented by the weighting error function vector $\overline{\overline{\mathbf{u}}}$. The smaller $\overline{\overline{\mathbf{u}}}$ is, the lower ISR will be obtained. From the definition of $\overline{\overline{\mathbf{u}}}$, the problem of reference signal selection can be stated as how to select a $K \times K$ matrix $\mathbf{A}_{r}$ from the given $N \times K$ matrix A to minimize $\overline{\overline{\mathbf{u}}}$. Since both $\mathbf{A}^{H} \mathbf{h}_{m}(l)$ and $\mathbf{A}_{r}^{H} \mathbf{w}_{m}(l, t) e^{j 2 \pi f_{c} l T_{d}}$ result in two vectors in the same space $\mathbb{C}^{K}$, the solution of this problem always exist. However, as $\mathbf{w}_{m}(l, t)$ is unknown, it is unable to find the solution using conventional least square approach. Instead, we propose an algorithm to find a sub-optimal solution as follows.

Defining $\Omega=\operatorname{Qdiag}\left\{\frac{1}{1+\mu \lambda_{i}}\right\} \mathbf{Q}^{-1}, i=1, \ldots, M \times K \times L$, from $\overline{\overline{\mathbf{u}}}=\boldsymbol{\Omega} \underline{\mathbf{h}}$, we see that $\boldsymbol{\Omega}$ is the transformation matrix of vector $\underline{\mathbf{h}}$, and $|\operatorname{det}(\Omega)|$ is the scaling factor of this transformation. As 
an example, Fig. 3 shows the transformation from $\underline{\mathbf{h}}$ to $\overline{\overline{\mathbf{u}}}$ when they are in the two dimensional space. The area of the rectangular defined by $\overline{\overline{\mathbf{u}}}$ (green) is equal to that of the rectangular defined by $\underline{\mathbf{h}}$ (orange) multiplied by $|\operatorname{det}(\Omega)|$. Therefore, $\mathbf{A}_{r}$ can be selected based on $|\operatorname{det}(\Omega)|$. From $\operatorname{det}(\boldsymbol{\Omega})=\prod_{i=1}^{M \times K \times L} \frac{1}{1+\mu \lambda_{i}}, \mathbf{A}_{r}$ can be found if the eigenvalues of the matrix $\boldsymbol{\Psi}$ satisfy that

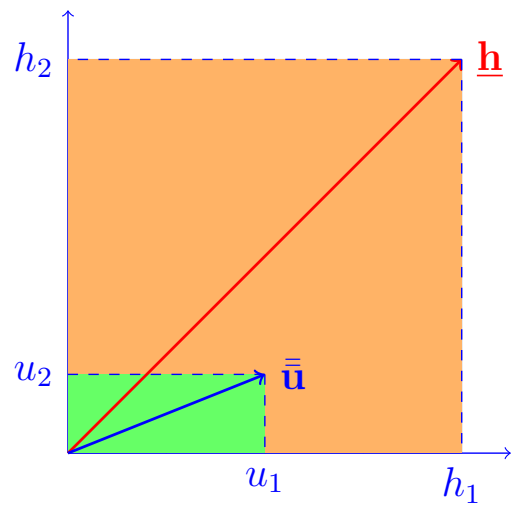

Fig. 3. Transformation of $\mathbf{h}$ to $\mathbf{u}$

$D_{\Omega}=\left|\prod_{i=1}^{M \times K \times L}\left(1+\mu \lambda_{i}\right)\right|$ is maximized. The solution found in this way is sub-optimal because there may be several selections of $\mathbf{A}_{r}$ that give the same $D_{\Omega}$, and $I S R$ is determined by the modulus of $\overline{\overline{\mathbf{u}}}$ rather than its volume. However, this is a simple method and the solution will always be found even though A may not be full ranked. The algorithm to select the reference signals is given in Algorithm 1 and is described as follows. Since $\mathbf{A}_{r}$ is formed by a combination of $K$ rows from $N$ rows in the matrix $\mathbf{A}$, there are $\left(\begin{array}{l}N \\ K\end{array}\right)$ possible selections which are indexed as Rind. $\mathbf{A}_{r}$ and $\Psi$ are then constructed, and $D_{\Omega}$ is calculated for all $\left(\begin{array}{l}N \\ K\end{array}\right)$. The sub-optimal $\mathbf{A}_{r}$ is found if it gives the highest $D_{\Omega}$. In Fig. 1, Algorithm 1 can be run in the reference signal selection block in the digital domain. From the users' beam angle information and transmitted signal autocorrelation matrix, the reference signals are determined and then connected to the cancellation circuits by the corresponding RF switches.

As an example, consider an $8 \times 8$ beamforming FD systems with 4 distinguished beams at angles $\theta_{k}=k \pi / 8, k=1, \ldots, 4$. All SI channels have 8 paths each with the path delay being the same as the tap delay in the ALMS loops. From 8 transmitted signals, there are 70 possible selections of the reference signals. Figure 4(a) presents the geometric mean of $D_{\Omega}$ calculated for all possible selections of $\mathbf{A}_{r}$ as well as the case of $\mathbf{A}_{r}=\mathbf{I}_{K}$. We can see that the maximum 


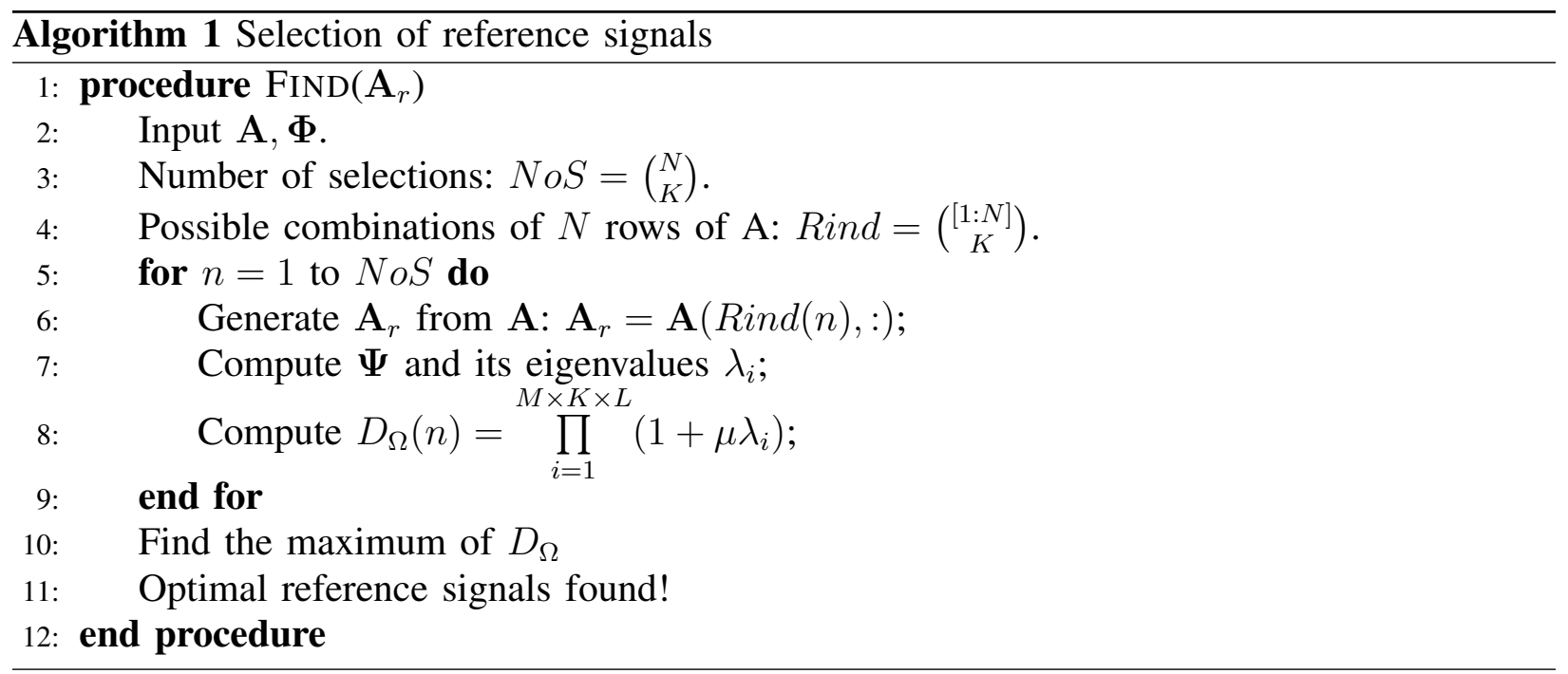

of $D_{\Omega}$ appears at $n=22$ and $n=30 . \mathbf{A}_{r}$ constructed at either these two positions is acceptable because the difference of $I S R \mathrm{~s}$ given by them is negligible as can be seen in Fig. 4(b). It is also worth noting that the optimal selection of the reference signals in the proposed structure provides higher level of cancellation than the case with additional Tx chains. This is because the beamforming matrix has an impact on the autocorrelation function of the transmitted signals so that the level of cancellation can be improved.

\section{Simulations AND COMPARISONS}

\section{A. Simulation Results}

Simulations are performed to illustrate the behaviors of the proposed cancellation structure. In all simulations, an $8 \times 8$ beamforming FD MIMO system communicates with four users at angles of $\theta_{k}=k \pi / 8, k=1, \ldots, 4$. The antenna elements are spaced at $30 \mathrm{~mm}$ which is a half of the wave-length of the transmitted carrier frequency $f_{c}=5 \mathrm{GHz}$. Data for all users are independent and QPSK modulated with symbol duration of $T_{s}=20 \mathrm{~ns}$. In all Tx chains, root-raised cosine pulse shaping filters with roll-off factor $\beta=0.25$ are employed. The power of the transmitted signal per antenna is $0 \mathrm{dBm}$ for $50 \mathrm{Ohm}$ load. The transmitted signals at the outputs of 8 antennas are labeled from 1 to 8 for reference signal selections. On the receiver side, the LNA gain $2 \mu$ in each $\mathrm{Rx}$ chain is selected as $\mu=10$. The ALMS loops are designed with $L=8$ taps equally spaced at $T_{d}=T_{s} / 2$. The multiplier dimensional constants are set at 


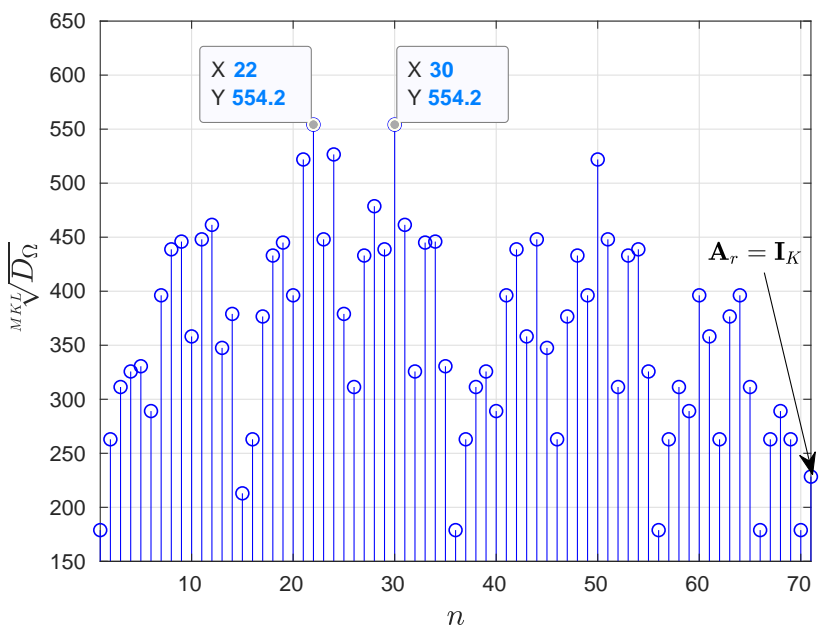

(a)

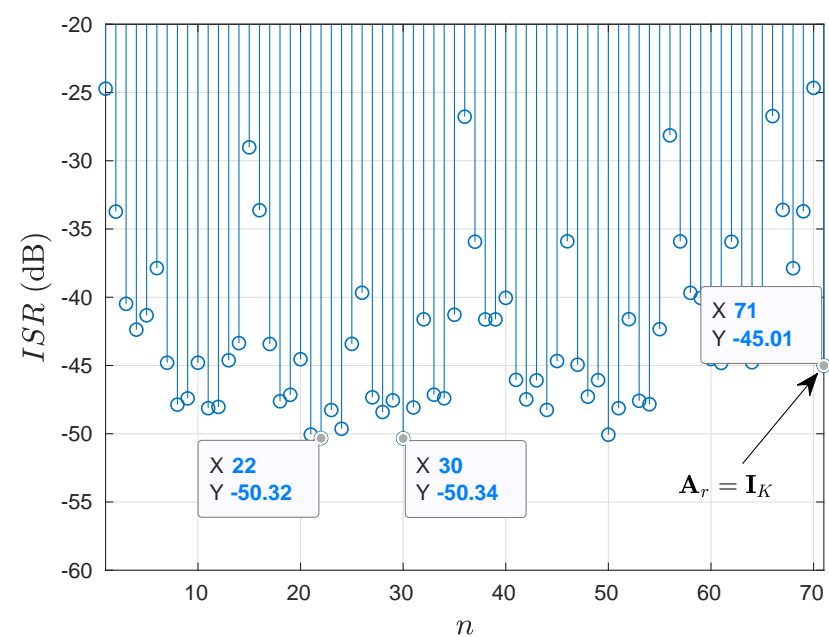

(b)

Fig. 4. (a) Geometric mean of $D_{\Omega}$ and (b) $I S R$ for all possible selections of $\mathbf{A}_{r}$

$K_{1} K_{2}=0.001 V^{2}$. The LPF parameter of all ALMS loop is chosen as $\alpha T_{s}=1.7655 \times 10^{-5}$ which is the same as in [13]. The propagation losses of all SI channels are set to be $25 \mathrm{~dB}$. In the first two simulations, the reference signals are chosen based on Algorithm 1, which are coupled at the outputs of Tx chains $1,3,5$ and 8 .

In the first simulation, the SI channel between a pair of $\mathrm{Tx}$ and $\mathrm{Rx}$ antennas is modeled with $L=8$ paths and the multipaths have an arbitrary delay. Specifically, the delay of the $l$-th path is chosen as $(l-1.5+$ rand $) T_{d}$ where rand is an uniformly distributed random number between 0 to 1 . It means that the maximum delay experienced by the SI signals is limited to be smaller than $L T_{d}$ which is the delay range covered by the ALMS loops. Fig. 5(a) presents 

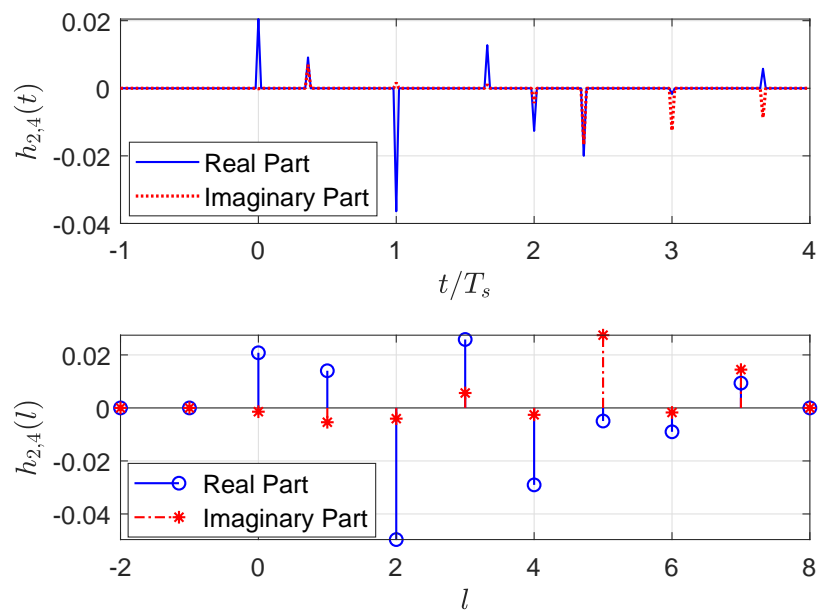

(a)
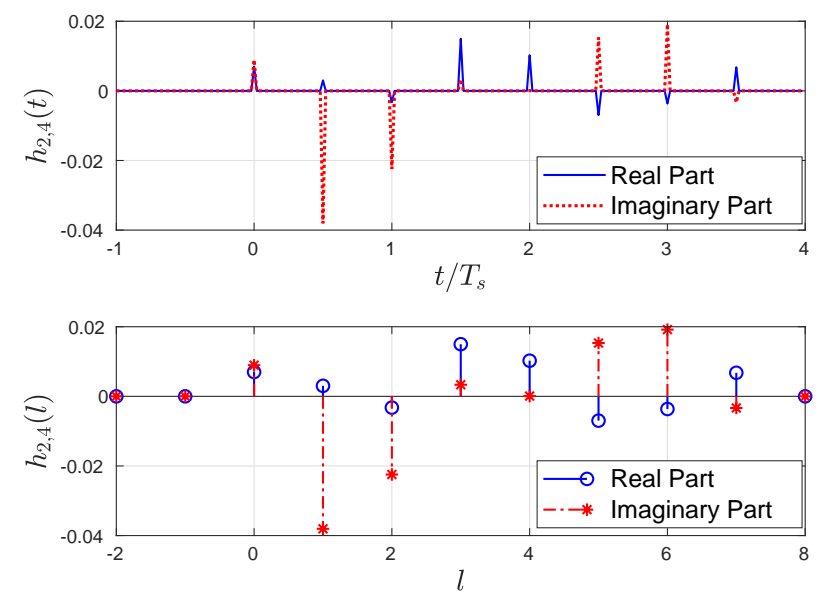

(b)

Fig. 5. SI channel impulse response $h_{2,4}(t)$ (top) and modeled tap coefficients $h_{2,4}(l)$ (bottom) for (a) arbitrary and (b) $T_{d}$ spaced delay between multipaths

the impulse response (top) and the modeled tap coefficients (bottom) of the SI channel from the fourth transmit antenna to the second received antenna. The modeling error calculated from (15) in this case is 0.002 . In the second simulation, the SI channels for all pairs of Tx and Rx antennas are modeled to have the same number of paths and path delay as in the ALMS loop so that the modeling error is zero. The channel impulse response and the modeled channel coefficients for the same SI channel in the first simulation are presented in Fig. 5(b).

Results of these two simulations are presented in Fig. 6. It can be seen in both simulations that the simulated results match the theoretical $I S R(t)$ (obtained from (23)) and hence confirm 


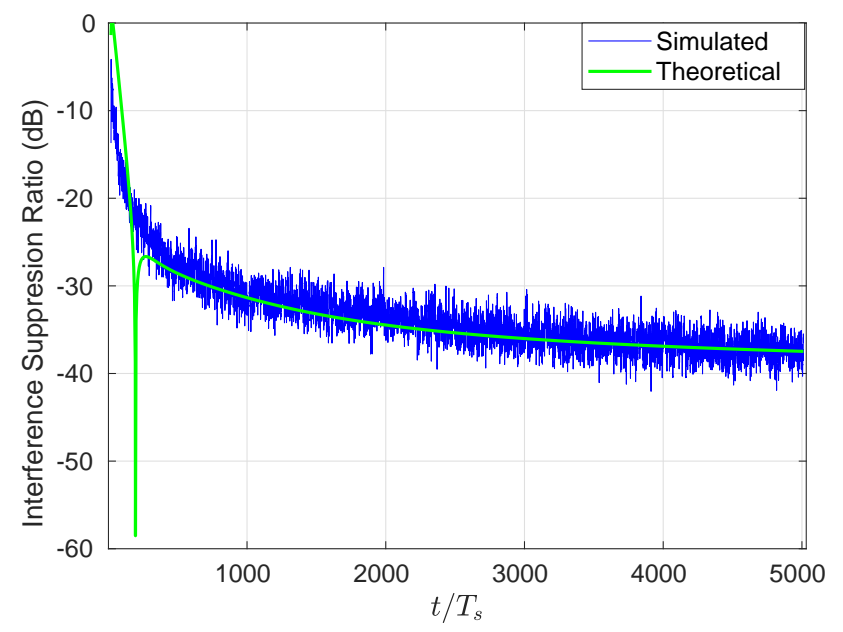

(a)

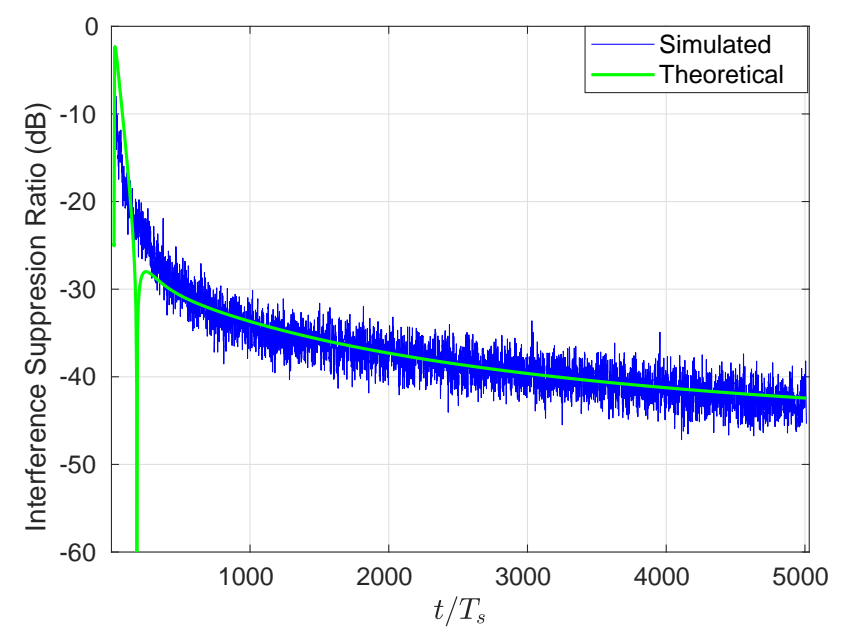

(b)

Fig. 6. Interference suppression ratio under (a) fractional interference channel and (b) $T_{d}$ spaced interference chanel.

the analyses presented in Section III. Due to the modeling error in the first simulation, the level of cancellation given by the ALMS loops is about $5 \mathrm{~dB}$ less than that in the second simulation. However, a level of $38 \mathrm{~dB}$ cancellation is still achieved.

In the third simulation, the reference signals are selected from the first four transmitted signals while the SI channels are modeled in the same way as in the second simulation. The simulation result for this case is presented in Fig. 7. We can see that even though the ALMS loop has exactly the same tap delay as in the SI channel, the performance of the ALMS loop is worse than that in the first simulation. These results demonstrate the importance of the selection of reference signals on the performance of the ALMS loop. 


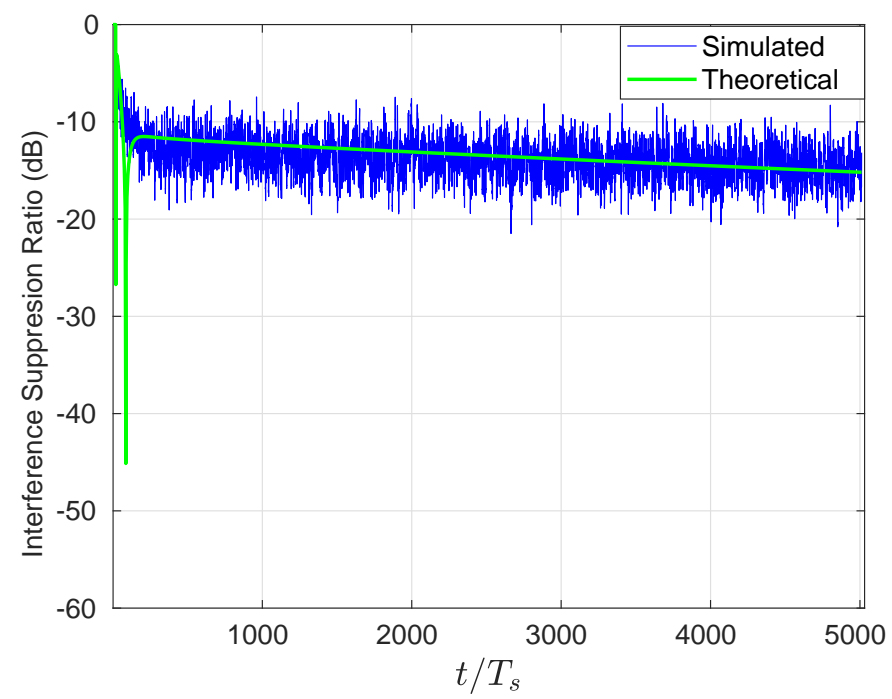

Fig. 7. Interference suppression ratio with the worst reference signals.

TABLE I

COMPARISON WITH EXISTING METHODS FOR FD MIMO

\begin{tabular}{|c|c|c|c|c|c|}
\hline \multirow{2}{*}{} & \multicolumn{3}{|c|}{ Complexity } & \multirow{2}{*}{ ISR } & \multirow{2}{*}{ Comments } \\
\cline { 2 - 4 } & Analog circuits & DSP & CSI & (dB) & \\
\hline$[24]$ & O(ML) & Involved & Required & 40 & $3 \times 3$ MIMO \\
\hline$[28]$ & O(MNL) & Involved & Required & 40 & $2 \times 2$ MIMO \\
\hline [29], [38], [39] & O(M)+MUX/DEMUX & Involved & Required & N/A & $4 \times 4$ MIMO \\
\hline Proposed & O(MKL $)$ & No & No & $38-42$ & $8 \times 8$ MIMO \\
\hline
\end{tabular}

\section{B. Comparison with existing methods for FD MIMO systems}

We compare the complexity and performance of the proposed structure with those of some other existing RF domain cancellation methods [24], [28], [29], [38], [39] as summarized in Table I. In this comparison, the complexity of each work includes the analog circuits, the requirement of CSI, and the involvement of DSP for tuning the cancellation circuits. The performance of these methods in terms of ISR is also provided for information and reference purpose since these FD MIMO systems have different number of antennas and transmission bandwidths. In addition, some baselines [29], [38], [39] present the sum-rate rather than ISR. As can be seen in Table I, all other baselines require DSP and CSI for tuning analog cancellation circuits. 


\section{CONCLUSION}

In this paper, we have proposed a novel beam-based analog SI cancellation architecture, which significantly reduces both hardware and signal processing complexities conventionally required for SI mitigation in FD MIMO systems. Considering interference at the receiver side as a linear combination of the data transmitted from all beams, we have proved that the number of adaptive filters at each Rx chain is reduced to the number of transmitted beams rather than as high as the number of Tx antennas. Further, a novel method has been proposed to select the optimized reference signals for the adaptive filters from the Tx antennas. Stationary analysis shows that, the level of SI cancellation given by the proposed structure is higher than that obtained by employing ALMS loops for the same number of adaptive filters with reference signals generated by additional Tx chains. Our future works will be the consideration of SI cancellation for FD hybrid beamforming MIMO systems and derivation of an optimal precoding matrix that enhances both SI cancellation and beamforming performance.

\section{ACKNOWLEDGMENT}

This work was supported by the Australian Research Council (DP160101693).

\section{APPENDIX A}

\section{DERIVATION OF MODELING ERROR}

From (13), we have

$$
\sum_{n^{\prime}=1}^{N} \int_{-\infty}^{\infty} h_{n^{\prime}, m}(\tau) \bar{E}\left\{X_{n}\left(t-l T_{d}\right) X_{n^{\prime}}^{*}(t-\tau)\right\} d \tau-\sum_{n^{\prime}=1}^{N} \sum_{l^{\prime}=0}^{L-1} h_{n^{\prime}, m}\left(l^{\prime}\right) \bar{E}\left\{X_{n}\left(t-l T_{d}\right) X_{n^{\prime}}^{*}\left(t-l^{\prime} T_{d}\right)\right\}=0
$$

for $n=0, \ldots, N$, and $l=0, \ldots, L-1$. Substituting $X_{n}(t)=\mathbf{a}_{n} \mathbf{S}(t)$ where $\mathbf{a}_{n}=\left[a_{n, 1}, \ldots, a_{n, K}\right]$ is the $n$-th row vector of $\mathbf{A}$ into (26), we get

$$
\begin{aligned}
& \sum_{n^{\prime}=1}^{N} \int_{-\infty}^{\infty} h_{n^{\prime}, m}(\tau) \mathbf{a}_{n} \bar{E}\left\{\mathbf{S}\left(t-l T_{d}\right) \mathbf{S}^{H}(t-\tau)\right\} \mathbf{a}_{n^{\prime}}^{H} d \tau \\
& \left.-\sum_{n^{\prime}=1}^{N} \sum_{l^{\prime}=0}^{L-1} h_{n^{\prime}, m}\left(l^{\prime}\right) \mathbf{a}_{n} \bar{E}\left\{\mathbf{S}\left(t-l T_{d}\right) \mathbf{S}^{H}\left(t-l^{\prime} T_{d}\right)\right\} \mathbf{a}_{n^{\prime}}^{H}\right\}=0 .
\end{aligned}
$$


From $E\left\{S_{k}(t) S_{k^{\prime}}(t-\tau)\right\}=0$ for $k \neq k^{\prime}$, (27) becomes

$$
\sum_{n^{\prime}=1}^{N}\left[\int_{-\infty}^{\infty} h_{n^{\prime}, m}(\tau) \Phi\left(l T_{d}-\tau\right) \mathbf{a}_{n} \mathbf{a}_{n^{\prime}}^{H} d \tau-\sum_{l^{\prime}=0}^{L-1} h_{n^{\prime}, m}\left(l^{\prime}\right) \Phi\left(\left(l-l^{\prime}\right) T_{d}\right) \mathbf{a}_{n} \mathbf{a}_{n^{\prime}}^{H}\right]=0 .
$$

Therefore,

$$
\int_{-\infty}^{\infty} h_{n, m}(\tau) \Phi\left(l T_{d}-\tau\right) d \tau=\sum_{l^{\prime}=0}^{L-1} h_{n, m}\left(l^{\prime}\right) \Phi\left(\left(l-l^{\prime}\right) T_{d}\right)
$$

or, in the matrix form

$$
\left[\begin{array}{c}
\int_{-\infty}^{\infty} \Phi(-\tau) h_{n^{\prime}, m}(\tau) d \tau \\
\vdots \\
\int_{-\infty}^{\infty} \Phi\left((L-1) T_{d}-\tau\right) h_{n^{\prime}, m}(\tau) d \tau
\end{array}\right]=\Phi\left[\begin{array}{c}
h_{n^{\prime}, m}(0) d \tau \\
\vdots \\
h_{n^{\prime}, m}(L-1) d \tau
\end{array}\right]
$$

Extend (30) to the full form of $N \times M$ channel, we found the vector of modeled channel coefficients $\mathbf{h}$ as

$$
\mathbf{h}=\left[\begin{array}{c}
h_{1,1}(0) \\
\vdots \\
h_{N, M}(L-1)
\end{array}\right]=\left[\mathbf{I}_{N M} \otimes \boldsymbol{\Phi}\right]^{-1}\left[\begin{array}{c}
\int_{-\infty}^{\infty} \Phi(-\tau) h_{1,1}(\tau) d \tau \\
\vdots \\
\int_{-\infty}^{\infty} \Phi\left((L-1) T_{d}-\tau\right) h_{N, M}(\tau) d \tau
\end{array}\right]
$$

The modeling error for SI channels from $\mathrm{N}$ Tx antennas to the $m$-th $\mathrm{Rx}$ antenna is calculated by

$$
\begin{aligned}
\epsilon_{m}^{2}= & \frac{1}{K_{1} K_{2}} \bar{E}\left\{\left|\sum_{n=1}^{N} \int_{-\infty}^{\infty} h_{n, m}^{*}(\tau) X_{n}(t-\tau) d \tau-\sum_{n=1}^{N} \sum_{l=0}^{L-1} h_{n, m}^{*}(l) X_{n}\left(t-l T_{d}\right)\right|^{2}\right\} \\
= & \frac{1}{K_{1} K_{2}} \bar{E}\left\{\left[\sum_{n=1}^{N} \int_{-\infty}^{\infty} h_{n, m}^{*}(\tau) X_{n}(t-\tau) d \tau-\sum_{n=1}^{N} \sum_{l=0}^{L-1} h_{n, m}^{*}(l) X_{n}\left(t-l T_{d}\right)\right]\right. \\
& \left.\cdot\left[\sum_{n^{\prime}=1}^{N} \int_{-\infty}^{\infty} h_{n^{\prime}, m}\left(\tau^{\prime}\right) X_{n^{\prime}}^{*}\left(t-\tau^{\prime}\right) d \tau^{\prime}-\sum_{n^{\prime}=1}^{N} \sum_{l^{\prime}=0}^{L-1} h_{n^{\prime}, m}\left(l^{\prime}\right) X_{n^{\prime}}^{*}\left(t-l^{\prime} T_{d}\right)\right]\right\} .
\end{aligned}
$$


From (26), (32) becomes

$$
\begin{aligned}
\epsilon_{m}^{2} & =\sum_{n=1}^{N} \sum_{n^{\prime}=1}^{N} \int_{-\infty}^{\infty} \int_{-\infty}^{\infty} h_{n, m}^{*}(\tau) \Phi\left(\tau-\tau^{\prime}\right) \mathbf{a}_{n} \mathbf{a}_{n^{\prime}}^{H} h_{n^{\prime}, m}\left(\tau^{\prime}\right) d \tau d \tau^{\prime} \\
& -\sum_{n=1}^{N} \sum_{n^{\prime}=1}^{N} \sum_{l^{\prime}=0}^{L-1} \int_{-\infty}^{\infty} h_{n, m}^{*}(\tau) \Phi\left(\tau-l^{\prime} T_{d}\right) \mathbf{a}_{n} \mathbf{a}_{n^{\prime}}^{H} h_{n^{\prime}, m}\left(l^{\prime}\right) d \tau \\
& =\sum_{n=1}^{N} \sum_{n^{\prime}=1}^{N} \int_{-\infty}^{\infty} \int_{-\infty}^{\infty} h_{n, m}^{*}(\tau) \Phi\left(\tau-\tau^{\prime}\right) \mathbf{a}_{n} \mathbf{a}_{n^{\prime}}^{H} h_{n^{\prime}, m}\left(\tau^{\prime}\right) d \tau d \tau^{\prime} \\
& -\sum_{n=1}^{N} \sum_{n^{\prime}=1}^{N} \sum_{l^{\prime}=0}^{L-1} \sum_{l=0}^{L-1} h_{n, m}^{*}(l) \Phi\left(\left(l-l^{\prime}\right) T_{d}\right) \mathbf{a}_{n} \mathbf{a}_{n^{\prime}}^{H} h_{n^{\prime}, m}\left(l^{\prime}\right) \\
& =\sum_{n=1}^{N} \sum_{n^{\prime}=1}^{N} \int_{-\infty}^{\infty} \int_{-\infty}^{\infty} h_{n, m}^{*}(\tau) \Phi\left(\tau-\tau^{\prime}\right) \mathbf{a}_{n} \mathbf{a}_{n^{\prime}}^{H} h_{n^{\prime}, m}\left(\tau^{\prime}\right) d \tau d \tau^{\prime}-\mathbf{h}_{m}^{H}\left[\mathbf{A} \otimes \mathbf{I}_{L}\right] \boldsymbol{\Theta}\left[\mathbf{A}^{H} \otimes \mathbf{I}_{L}\right] \mathbf{h}_{m}
\end{aligned}
$$

where $\mathbf{h}_{m}=\left[h_{1, m}(0), \ldots, h_{N, m}(L-1)\right]^{T}$.

\section{APPENDIX B}

\section{DERIVATION OF WEIGHTING ERROR FUNCTIONS}

From (6), we have

$$
\begin{aligned}
\mathbf{w}_{m}\left(l, t+t_{0}\right) & =\frac{2 \mu \alpha}{K_{1} K_{2}} \int_{t_{0}}^{t+t_{0}} e^{-\alpha\left(t+t_{0}-\tau\right)} \operatorname{Re}\left\{\left[D_{m}(\tau)+S_{m, r}(\tau)+N_{m}(\tau)\right] e^{j 2 \pi f_{c} \tau}\right\} \\
& \cdot \mathbf{X}_{r}\left(\tau-l T_{d}\right) e^{j 2 \pi f_{c}\left(\tau-l T_{d}\right)} d \tau \\
& =\frac{\mu \alpha}{K_{1} K_{2}} \int_{t_{0}}^{t+t_{0}} e^{-\alpha\left(t+t_{0}-\tau\right)}\left\{\left[D_{m}(\tau)+S_{m, r}(\tau)+N_{m}(\tau)\right] e^{j 2 \pi f_{c} \tau}\right. \\
& \left.+\left[D_{m}^{*}(\tau)+S_{m, r}^{*}(\tau)+N_{m}^{*}(\tau)\right] e^{-j 2 \pi f_{c} \tau}\right\} \mathbf{X}_{r}\left(\tau-l T_{d}\right) e^{j 2 \pi f_{c}\left(\tau-l T_{d}\right)} d \tau
\end{aligned}
$$

where $D_{m}(t)=\sum_{l=0}^{L-1} \mathbf{h}_{m}^{H}(l) \mathbf{X}\left(t-l T_{d}\right)-\sum_{l=0}^{L-1} e^{-j 2 \pi f_{c} l T_{d}} \mathbf{w}_{m}^{H}(l, t) \mathbf{X}_{r}\left(t-l T_{d}\right)$ is the low-pass equivalent of the residual SI signal $d_{m}(t)=z_{m}(t)-y_{m}(t)$. Substituting $\mathbf{X}(t)=\mathbf{A S}(t)$ and $\mathbf{X}_{r}(t)=\mathbf{A}_{r} \mathbf{S}(t)$ into the expression of $D_{m}(t)$ and noting that $D_{m}(t)$ is a scalar function (hence 
$\left.D_{m}^{T}(t)=D_{m}(t)\right)$, we obtain

$$
D_{m}^{*}(t)=\sum_{l=0}^{L-1} \mathbf{S}^{H}\left(t-l T_{d}\right)\left[\mathbf{A}^{H} \mathbf{h}_{m}(l)-\mathbf{A}_{r}^{H} e^{j 2 \pi f_{c} l T_{d}} \mathbf{w}_{m}(l, t)\right]
$$

Substituting (35) into (34), after some multiplications and noting that signal components centered about the frequency $2 f_{c}$ are eliminated by the LPF whose bandwidth is assumed to be very narrow compared to $f_{c}$, we get

$$
\begin{aligned}
\mathbf{w}_{m}\left(l, t+t_{0}\right) & =\frac{\mu \alpha}{K_{1} K_{2}} \int_{t_{0}}^{t+t_{0}} e^{-\alpha\left(t+t_{0}-\tau\right)}\left\{\sum_{l^{\prime}=0}^{L-1} \mathbf{S}^{H}\left(\tau-l^{\prime} T_{d}\right)\left[\mathbf{A}^{H} \mathbf{h}_{m}\left(l^{\prime}\right)-\mathbf{A}_{r}^{H} e^{j 2 \pi f_{c} l^{\prime} T_{d}} \mathbf{w}_{m}\left(l^{\prime}, \tau\right)\right]\right. \\
& \left.+S_{r}^{*}(\tau)+N^{*}(\tau)\right\} \mathbf{A}_{r} e^{-j 2 \pi f_{c} l T_{d}} \mathbf{S}\left(\tau-l T_{d}\right) d \tau
\end{aligned}
$$

Multiplying both sides of (36) with $\mathbf{A}_{r}^{H} e^{j 2 \pi f_{c} l T_{d}}$ and defining $\mathbf{B}=\mathbf{A}_{r}^{H} \mathbf{A}_{r}$, we have

$$
\begin{array}{r}
\mathbf{A}_{r}^{H} e^{j 2 \pi f_{c} l T_{d}} \mathbf{w}_{m}\left(l, t+t_{0}\right)=\frac{\mu \alpha}{K_{1} K_{2}} \int_{t_{0}}^{t+t_{0}} e^{-\alpha\left(t+t_{0}-\tau\right)}\left\{\sum _ { l ^ { \prime } = 0 } ^ { L - 1 } \mathbf { S } ^ { H } ( \tau - l ^ { \prime } T _ { d } ) \left[\mathbf{A}^{H} \mathbf{h}_{m}\left(l^{\prime}\right)-\right.\right. \\
\left.\left.\mathbf{A}_{r}^{H} e^{j 2 \pi f_{c} l^{\prime} T_{d}} \mathbf{w}_{m}\left(l^{\prime}, \tau\right)\right]+S_{r}^{*}(\tau)+N^{*}(\tau)\right\} \mathbf{B S}\left(\tau-l T_{d}\right) d \tau .
\end{array}
$$

Substituting the definition of $\mathbf{u}_{m}(l, t)=\mathbf{A}^{H} \mathbf{h}_{m}(l)-\mathbf{A}_{r}^{H} e^{j 2 \pi f_{c} l T_{d}} \mathbf{w}_{m}(l, \tau)$ into (37), we have

$$
\begin{aligned}
\mathbf{u}_{m}\left(l, t+t_{0}\right) & =\mathbf{A}^{H} \mathbf{h}_{m}(l)-\frac{\mu \alpha}{K_{1} K_{2}} \int_{t_{0}}^{t+t_{0}} e^{-\alpha\left(t+t_{0}-\tau\right)}\left[\sum_{l^{\prime}=0}^{L-1} \mathbf{S}^{H}\left(\tau-l^{\prime} T_{d}\right) \mathbf{u}_{m}\left(l^{\prime}, \tau\right)\right. \\
& \left.+S_{r}^{*}(\tau)+N^{*}(\tau)\right] \mathbf{B S}\left(\tau-l T_{d}\right) d \tau
\end{aligned}
$$

Taking ensemble expectation on two sides of (38) and assuming that all the transmitted signals are independent to the received signals and noise, we have

$$
\overline{\mathbf{u}}_{m}\left(l, t+t_{0}\right)=\mathbf{A}^{H} \mathbf{h}_{m}(l)-\frac{\mu \alpha}{K_{1} K_{2}} \int_{t_{0}}^{t+t_{0}} e^{-\alpha\left(t+t_{0}-\tau\right)} \sum_{l^{\prime}=0}^{L-1} \mathbf{B S}\left(\tau-l^{\prime} T_{d}\right) \mathbf{S}^{H}\left(\tau-l T_{d}\right) \overline{\mathbf{u}}_{m}\left(l^{\prime}, \tau\right) d \tau .
$$


Taking time average over one symbol period $T_{s}$ of $\overline{\mathbf{u}}_{m}\left(l, t+t_{0}\right)$ with respect to the starting time $t_{0}$, we obtain

$$
\overline{\overline{\mathbf{u}}}_{m}(l, t)=\mathbf{A}^{H} \mathbf{h}_{m}(l)-\mu \alpha \int_{0}^{t} e^{-\alpha(t-\tau)} \sum_{l^{\prime}=0}^{L-1} \mathbf{B \Theta}\left(\left(l-l^{\prime}\right) T_{d}\right) \overline{\mathbf{u}}_{m}\left(l^{\prime}, \tau\right) d \tau
$$

where $\Theta\left(\left(l-l^{\prime}\right) T_{d}\right)=\Phi\left(\left(l-l^{\prime}\right) T_{d}\right) \mathbf{I}_{K}$. In derivation of (40), we have assumed that $\overline{\mathbf{u}}_{m}(l, t)$ changes slowly and it can be seen as a constant during $T_{s}$, i.e., $\overline{\mathbf{u}}_{m}\left(l, t+t_{0}\right) \approx \overline{\overline{\mathbf{u}}}_{m}(l, t)=$ $\frac{1}{T_{s}} \int_{0}^{T_{s}} \overline{\mathbf{u}}_{m}\left(l, t+t_{0}\right) d t_{0}$. Eq. (40) can be rewritten in the full form of $L$-taps as

$$
\overline{\overline{\mathbf{u}}}_{m}(t)=\left(\mathbf{A}^{H} \otimes \mathbf{I}_{L}\right) \mathbf{h}_{m}-\mu \alpha \int_{0}^{t} e^{-\alpha(t-\tau)}\left[\left(\mathbf{B} \otimes \mathbf{I}_{L}\right) \Theta\right] \overline{\overline{\mathbf{u}}}_{m}(\tau) d \tau .
$$

Expanding (41) to all $M \mathrm{Rx}$ chains, we obtain the expected weighting error function vector as

$$
\overline{\overline{\mathbf{u}}}(t)=\left[\mathbf{I}_{M} \otimes\left(\mathbf{A}^{H} \otimes \mathbf{I}_{L}\right)\right] \mathbf{h}-\mu \alpha \int_{0}^{t} e^{-\alpha(t-\tau)}\left\{\mathbf{I}_{M} \otimes\left[\left(\mathbf{B} \otimes \mathbf{I}_{L}\right) \Theta\right]\right\} \overline{\overline{\mathbf{u}}}(\tau) d \tau .
$$

Denoting $\Psi=\mathbf{I}_{M} \otimes\left[\left(\mathbf{B} \otimes \mathbf{I}_{L}\right) \boldsymbol{\Theta}\right]$ and $\underline{\mathbf{h}}=\left[\mathbf{I}_{M} \otimes\left(\mathbf{A}^{H} \otimes \mathbf{I}_{L}\right)\right] \mathbf{h}$, we obtain the final equation of $\overline{\overline{\mathbf{u}}}(t)$ as in $(21)$.

\section{REFERENCES}

[1] J. G. Andrews et al., "What will 5G be?” IEEE J. Sel. Areas Commun, vol. 32, no. 6, pp. 1065-1082, June 2014.

[2] F. Boccardi et al., "Five disruptive technology directions for 5G," IEEE Communications Magazine, vol. 52, no. 2, pp. 74-80, Feb. 2014.

[3] M. Agiwal, A. Roy, and N. Saxena, "Next generation 5G wireless networks: A comprehensive survey," vol. 18, no. 3, pp. $1617-1655,2016$.

[4] S. Goyal et al., "Full duplex cellular systems: will doubling interference prevent doubling capacity?" IEEE Communications Magazine, vol. 53, no. 5, pp. 121-127, May 2015.

[5] J. Koh et al., "On the feasibility of full-duplex large-scale MIMO cellular systems," IEEE Trans. Wireless Commun., vol. 17, no. 9, pp. 6231-6250, Sept. 2018.

[6] G. Zheng, "Joint beamforming optimization and power control for full-duplex MIMO two-way relay channel," IEEE Trans. Signal Process., vol. 63, no. 3, pp. 555-566, Feb. 2015.

[7] Z. Zhang et al., "Full-duplex wireless communications: Challenges, solutions, and future research directions," Proceedings of the IEEE, vol. 104, no. 7, pp. 1369-1409, July 2016. 
[8] J. Choi, M. Jain, and K. Srinivasan, “Achieving single channel, full duplex wireless communication,” in Proc. 16th Annu. Int. Conf. Mobi. Commun. Chicago, USA, Sept. 2010, pp. 1-12.

[9] M. Duarte et al., "Design and characterization of a full-duplex multiantenna system for WIFI networks," IEEE Trans. Veh. Technol., vol. 63, no. 3, pp. 1160-1177, Mar 2014.

[10] T. Oh et al., "Dual-polarization slot antenna with high cross-polarization discrimination for indoor small-cell MIMO systems," IEEE Antennas Wireless Propag. Lett., vol. 14, pp. 374-377, Oct. 2014.

[11] K. E. Kolodziej, J. G. McMichael, and B. T. Perry, "Multitap RF canceller for in-band full-duplex wireless communications," IEEE Trans. Wireless Commun., vol. 15, no. 6, pp. 4321-4334, June 2016.

[12] A. Kiayani et al., "Adaptive nonlinear RF cancellation for improved isolation in simultaneous transmit-receive systems," IEEE Trans. Microw. Theory Techn., vol. 66, no. 5, pp. 2299-2312, Jan. 2018.

[13] X. Huang and Y. J. Guo, "Radio frequency self-interference cancellation with analog least mean-square loop," IEEE Trans. Microw. Theory Tech., vol. 65, no. 9, pp. 3336-3350, Sept. 2017.

[14] D. Korpi et al., "Widely linear digital self-interference cancellation in direct-conversion full-duplex transceiver," IEEE J. Sel. Areas Commun, vol. 32, no. 9, pp. 1674-1687, Sept. 2014.

[15] E. Ahmed and A. M. Eltawil, "All-digital self-interference cancellation technique for full-duplex systems," IEEE Trans. Wireless Commun., vol. 14, no. 7, pp. 3519-3532, July 2015.

[16] D. Bharadia, E. McMilin, and S. Katti, "Full duplex radios," in Proc. ACM SIGCOMM 2013, Hong Kong, China, Aug. 2013, pp. 375-386.

[17] M. Chung et al., "Prototyping real-time full duplex radios," IEEE Communications Magazine, vol. 53, no. 9, pp. 56-63, Sept. 2015.

[18] D. W. Bliss, P. A. Parker, and A. R. Margetts, "Simultaneous transmission and reception for improved wireless network performance," in 2007 IEEE/SP 14th Workshop Statistical Signal Process., Aug 2007, pp. 478-482.

[19] T. Snow, C. Fulton, and W. J. Chappell, "Transmit-Receive duplexing using digital beamforming system to cancel selfinterference," IEEE Trans. Microw. Theory Techn., vol. 59, no. 12, pp. 3494-3503, Dec. 2011.

[20] T. Riihonen, S. Werner, and R. Wichman, "Mitigation of loopback self-interference in full-duplex MIMO relays," IEEE Trans. Signal Process, vol. 59, no. 12, pp. 5983-5993, Dec. 2011.

[21] E. Aryafar et al., "MIDU: Enabling MIMO full duplex," in Proc. Ann. Inter. Conf. Mobile Comput. Netw., ser. Mobicom '12. New York, NY, USA: ACM, 2012, pp. 257-268. [Online]. Available: http://doi.acm.org/10.1145/2348543.2348576

[22] E. Everett et al., "Softnull: Many-antenna full-duplex wireless via digital beamforming," IEEE Trans. Wireless Commun., vol. 15, no. 12, pp. 8077-8092, Dec. 2016.

[23] J. P. Doane, K. E. Kolodziej, and B. T. Perry, "Simultaneous transmit and receive performance of an 8-channel digital phased array," in 2017 IEEE Int. Sympo. Antennas Propag. USNC/URSI Nat. Radio Science Meeting, July 2017, pp. $1043-1044$.

[24] D. Bharadia and S. Katti, "Full duplex MIMO radios," in Proc. 11th USENIX Conf. Networked Systems Design Implementation, ser. NSDI'14. Berkeley, CA, USA: USENIX Association, 2014, pp. 359-372. [Online]. Available: http://dl.acm.org/citation.cfm?id=2616448.2616482 
[25] T. Huusari et al., "Wideband self-adaptive RF cancellation circuit for full-duplex radio: Operating principle and measurements," in Proc. 81st IEEE Veh. Technol. Conf. (VTC Spring), Glasgow, Scotland, 2015, pp. 11-14.

[26] L. Zhang, M. Ma, and B. Jiao, "Design and implementation of adaptive multi-tap analog interference canceller," IEEE Trans. Wireless Commun., vol. 18, no. 3, pp. 1698-1706, March 2019.

[27] K. E. Kolodziej, B. T. Perry, and J. S. Herd, "In-band full-duplex technology: Techniques and systems survey," IEEE Trans. Microw. Theory Techn., vol. 67, no. 7, pp. 3025-3041, July 2019.

[28] Y. Liu et al., "Multi-tap analog MIMO self-interference cancellation for full-duplex communications," in 2017 9th Int. Conf. Wireless Commun. Signal Process. (WCSP), Oct. 2017, pp. 1-6.

[29] G. C. Alexandropoulos and M. Duarte, "Joint design of multi-tap analog cancellation and digital beamforming for reduced complexity full duplex MIMO systems," in 2017 IEEE Int. Conf. Commun. (ICC), May 2017, pp. 1-7.

[30] H. Iimori and G. Thadeu Freitas de Abreu, "Two-way full-duplex MIMO with hybrid TX-RX MSE minimization and interference cancellation," in 2018 IEEE 19th Int. Workshop Signal Process. Advances Wireless Commun. (SPAWC), June 2018, pp. 1-5.

[31] A. T. Le, L. C. Tran, and X. Huang, "On performance of analog least mean square loop for self-interference cancellation in in-band full-duplex OFDM systems," in Proc. 85th IEEE Veh. Technol. Conf. (VTC Spring), Sydney, Australia, 2017, pp. 1-5.

[32] _ _ "Cyclostationary analysis of analog least mean square loop for self-interference cancellation in in-band full-duplex systems," IEEE Commun. Lett., vol. 21, no. 12, pp. 2738-2741, Sept. 2017.

[33] A. T. Le et al., "Analog least mean square loop for self-interference cancellation in generalized continuous wave SAR," in Proc. 88th IEEE Veh. Technol. Conf. (VTC Spring), Chicago, USA, 2018, pp. 1-5.

[34] — - "Frequency domain characterization and performance bounds of ALMS loop for RF self-interference cancellation," IEEE Trans. Commun., vol. 67, no. 1, pp. 682-692, Jan. 2019.

[35] A. T. Le et al., "Analog least mean square loop with I/Q imbalance for self-interference cancellation in full-duplex radios," IEEE Trans. Vehicular Technol., vol. pp, no. 99, pp. 1-13, 2019.

[36] _ _ "Analog least mean square loop for self-interference cancellation: Implementation and measurements," to appear in Proc. 29th Int. Telecommun. Networks Appl. Conf. (ITNAC), Aukland, New Zealand, Nov. 2019, pp. 1-5.

[37] A. T. Le et al., "Beam-based analog self-interference cancellation with auxiliary transmit chains in full-duplex MIMO systems," in 2019 IEEE 20th Int. Workshop Signal Process. Advances Wireless Commun. (SPAWC), July 2019, pp. 1-5.

[38] H. Iimori, G. Abreu, and G. C. Alexandropoulos, "Mimo beamforming schemes for hybrid sic fd radios with imperfect hardware and csi," IEEE Trans. Wireless Commun., pp. 1-1, 2019.

[39] M. A. Islam, G. C. Alexandropoulos, and B. Smida, "A unified beamforming and A/D self-interference cancellation design for full duplex mimo radios," in IEEE Int. Symp. Personal, Indoor Mobile Radio Commun., Sep 2019, pp. 1-5. 